\title{
Outcomes after gamma knife radiosurgery in solitary acoustic tumors and neurofibromatosis Type 2
}

\author{
Douglas Kondziolka, M.D., M.Sc., F.R.C.S.(C), Brian R. Subach, M.D., L. Dade Lunsford, M.D., \\ David J. Bissonette, P.A.C., M.B.A., and John C. Flickinger, M.D.
}

Departments of Neurological Surgery and Radiation Oncology, and the Center for Image-Guided Neurosurgery, University of Pittsburgh, Pittsburgh, Pennsylvania

Surgeons perform stereotactic radiosurgery as the main alternative to acoustic tumor (vestibular schwannoma) resection. The goals of radiosurgery include preservation of neurological function and prevention of tumor growth. Longer-term outcomes are not well documented for patients with solitary tumors or those with neurofibromatosis Type 2 (NF2).

To define outcomes, the authors evaluated 462 consecutive patients with solitary acoustic tumors and 40 patients with NF2 (total of 45 tumors treated) who underwent radiosurgery between 1987 and 1998.

Serial imaging studies, clinical evaluations, and a patient survey were performed. The average tumor margin dose was $15 \mathrm{~Gy}$, and the mean transverse tumor diameter was $22 \mathrm{~mm}$. In patients with solitary tumors, prior resection had been performed in 111 patients (24\%); 27 patients experienced tumor recurrence after a "total resection."

The clinical tumor control rate (no resection required) was $98 \%$. In non-NF2 patients followed for at least 5 years, 100 tumors $(61.7 \%)$ were smaller, 53 (32.7\%) remained unchanged in size, and nine (5.6\%) were slightly larger. Resection was performed in four patients $(2.4 \%)$. Neurological deficits after radiosurgery all occurred within the first 28 months. The rates of facial and trigeminal neuropathy varied with radiosurgery technique. In patients with NF2, 16 tumors were smaller, 28 remained unchanged, and one enlarged (overall $98 \%$ control rate at median 3-year follow up). Resection was performed in three patients (7\%). Useful hearing was preserved in six (43\%) of 14 NF2 patients who had useful hearing before radiosurgery.

Radiosurgery provided long-term tumor control associated with high rates of neurological function preservation. No further tumor surgery was necessary in $98 \%$ of patients with solitary tumors followed for a minimum of 5 years.

Key Words * acoustic neuroma * vestibular schwannoma * radiosurgery * hearing * facial nerve

The long-term outcomes after radiosurgery or microsurgery in patients with acoustic neuromas remain to 
be documented. Few authors report the use of serial imaging studies and clinical evaluations to determine outcomes and recurrence rates over time.[3,5,10,14,20,23-25] There are no reports documenting clinical outcomes that include patient employment status and satisfaction. Detailed yet early assessments of the results after gamma knife radiosurgery have been published.[3-7,20-23] Only preliminary information is available on results after radiosurgery for the management of neurofibromatosis Type 2 (NF2) tumors.[17] To define outcomes after acoustic neuroma radiosurgery, we studied all patients who underwent radiosurgery at a single center between 1987 and 1998. Early and later outcomes (5-10 years) were determined through the use of serial imaging studies, neurophysiological tests, and physician-based evaluations.

\section{CLINICAL MATERIAL AND METHODS}

\section{Patient Characteristics}

Five hundred and two patients underwent stereotactic radiosurgery for an acoustic tumor (vestibular schwannoma) at the University of Pittsburgh over an 11-year period. These included 462 patients with solitary tumors and 40 with NF2 tumors (45 tumors managed). Patient characteristics are listed in Table 1 .

\begin{tabular}{|c|c|c|}
\hline CHARACTERISTICS OF PATIENTS BEF & ONG ACOUSTIC & DOSURGERY \\
\hline Characteristic & $\begin{array}{l}462 \text { Non-N F2 } \\
\text { Patients ( } 96)\end{array}$ & $\begin{array}{c}40 \mathrm{NF} 2 \\
\text { Patients (\%) }\end{array}$ \\
\hline $\begin{array}{l}\text { age (yrs) } \\
\text { mean } \\
\text { range } \\
\text { female } \\
\text { male } \\
\text { prior subtotal tum or resection } \\
\text { prior gross-totil resection } \\
\text { prior fractionated radiation therapy } \\
\text { some hearing loss } \\
\text { normal facial ner we function } \\
\text { facial num bness } \\
\text { balanoe protlems } \\
\text { tinnitus } \\
\text { dysphagia }\end{array}$ & $\begin{array}{c}59 \\
24-84 \\
224(49) \\
238(51) \\
84(18) \\
27(6) \\
1(0.2) \\
415(90) \\
382(83) \\
94(00) \\
239(52) \\
183(40) \\
22(5)\end{array}$ & $\begin{array}{l}35 \\
12-79 \\
21(52) \\
19(48) \\
11(24) \\
2(4) \\
1(2.1) \\
42(93) \\
31(69) \\
9(20) \\
21(47) \\
17(38) \\
2(4)\end{array}$ \\
\hline
\end{tabular}

Prior resection had been performed in 111 patients (24\%). Twenty patients underwent two resections; five patients, three resections; and two patients, four or more resections. Although normal facial function (House-Brackmann Grade 1) was present in 382 patients (82.7\%), 27 patients had Grade 2 function; 20, Grade 3 function; 10, Grade 4 function; seven, Grade 5 function; and 16, Grade 6 function.[11] The Gardner-Robertson[9] scale was used to code hearing function. "Useful" hearing before radiosurgery was noted by $29 \%$ of patients.

Prior resection was performed in 15 patients with NF2. Two resections were performed in three patients and four resections in another three patients. Normal facial function before radiosurgery was present in $69 \%$, normal trigeminal function in $80 \%$, and useful hearing (Gardner-Robertson Grades 1 and 2) in $31 \%$. 
All patients underwent stereotactic radiosurgery in which the gamma knife (Elekta Instruments, Atlanta, GA) was used, supplemented with local anesthesia and intravenous sedation as necessary. Children younger than 12 years of age with NF2 underwent radiosurgery after induction of general anesthesia. Radiosurgery was guided by computerized tomography (CT) scanning during the period between 1987 and 1991. Subsequent patients underwent radiosurgery guided by magnetic resonance (MR) imaging after a prospective comparison study confirmed the accuracy of MR-based stereotactic targeting.[12] Multiple irradiation isocenters were used to fit the intracanalicular and extracanalicular tumor components.[8] The 50\% isodose line was used to cover the tumor margin in 401 patients (87\%) with solitary tumors. An initial tumor margin dose of 18 to $20 \mathrm{~Gy}$ was selected based on reported experience from the Karolinska group in Stockholm.[20] This dose was decreased to 16 to 18 Gy within the first 2 years and by 1992 was decreased further to a margin dose of 14 to $16 \mathrm{~Gy}$. Repeated evaluations of the cranial nerve response prompted small decreases in dose to improve preservation of cranial nerve function. $[7,13,15]$ The mean dose delivered to the margin of both non-NF2 and NF2 tumors was 15 Gy, and the mean maximum tumor dose was $29 \mathrm{~Gy}$. Dose selection in individual patients was based on the factors of tumor volume, surgical history, hearing status, facial motor function, and patient desires. After radiosurgery, patients received a single intravenous 40-mg dose of methylprednisolone and were discharged from the hospital the next morning.

\section{Follow-Up Evaluations}

Serial imaging studies (MR imaging or CT scanning when MR imaging was contraindicated) were obtained every 6 months for the first 2 years, annually for the next 2 years, and then bi-annually. Serial audiograms were obtained at 6- to 12-month intervals in patients with hearing. Contrast-enhanced imaging studies were performed to define the tumor response and to identify any peritumoral imaging changes or hydrocephalus. Before and after radiosurgery, each tumor was measured in five separate dimensions. (three extracanalicular and two intracanalicular) by using a previously reported method.[16] A significant imaging change using this caliper technique, was defined as a difference of $\pm 2 \mathrm{~mm}$.

\section{RESULTS}

\section{Imaging Response After Radiosurgery}

A comprehensive evaluation was made of patients with a minimum of 5 years of clinical follow up (162 patients). The details of this study will be reported elsewhere.[13] The results of all imaging studies were entered into a database and compared over time according to whether the tumor size was decreased, unchanged, or increased. The majority of irradiated acoustic tumors decreased in size over time (Fig. 1).[13] 


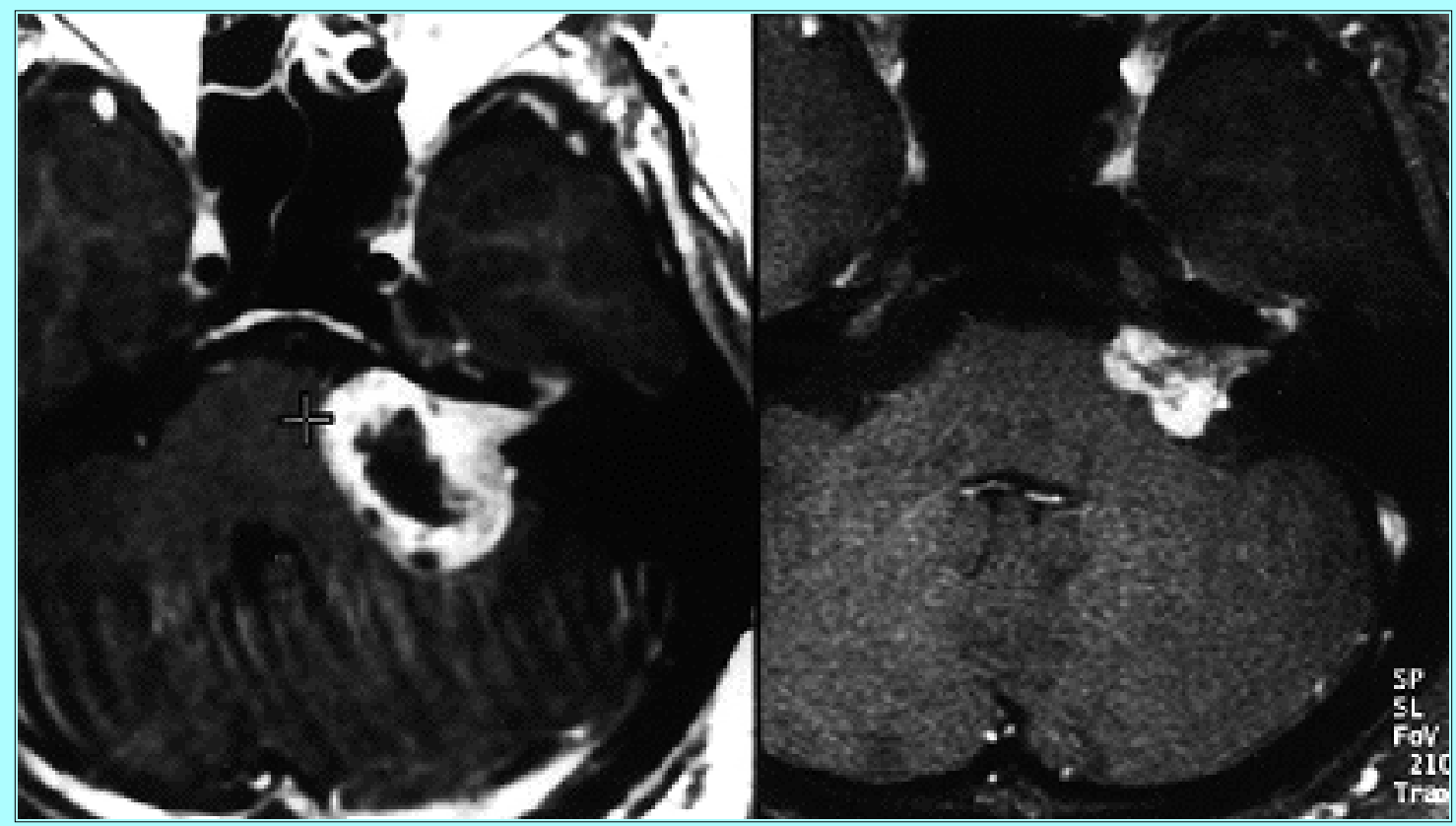

Fig. 1. Contrast-enhanced axial MR images showing a left acoustic tumor (cross) in a young man with the human immunodeficiency virus. Left: Preoperative image. Right: Three years after radiosurgery, major tumor regression can be identified without new neurological symptoms.

At the 1-year follow-up evaluation, only $26 \%$ of tumors had decreased in size but by the 3rd year, $59 \%$ of patients had smaller tumors. Nine patients had tumors that increased in size, and all were identified within the first 3 years after radiosurgery. Enlargement represented either true neoplastic tumor growth (four patients) or tumor death with an expansion of the tumor margins as the central portion of the tumor became necrotic (five patients). In the latter patients subsequent imaging studies confirmed tumor-volume regression. Four patients underwent resection. No further increase in tumor volume was identified in any patient during the period from 4 to 10 years after radiosurgery. Of patients evaluated between the 5 th and 10th years, $72 \%$ had a decrease in tumor volume and $28 \%$ had no change in tumor size (Fig. 2).
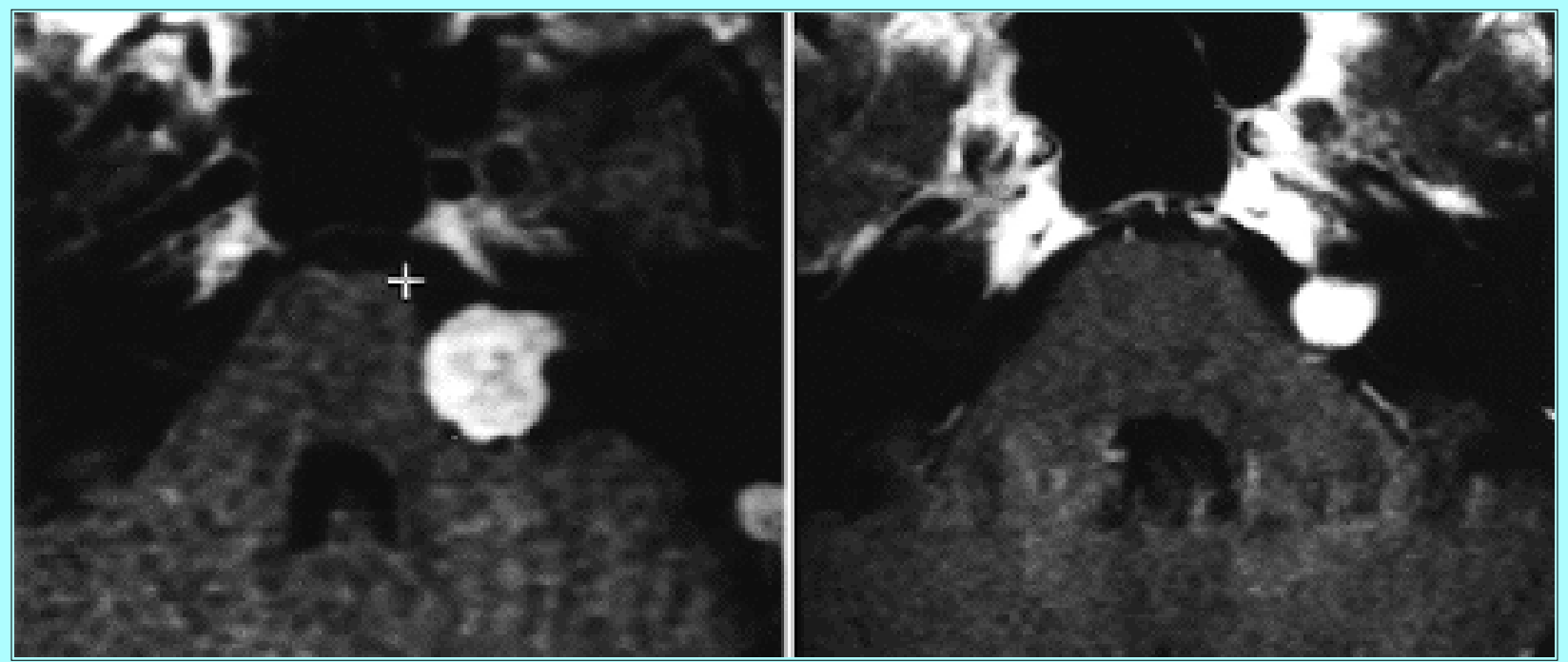

Fig. 2. Contrast-enhanced axial MR images showing a left acoustic tumor (cross) in a woman at the time of radiosurgery (left) and 6 years later (right). Significant tumor regression was noted, and the tumor no longer compressed the middle cerebellar peduncle. 


\section{Clinical Condition of the Patients}

A few patients experienced transient headache after removal of the stereotactic frame. There were no infections or systemic complications. Patients returned to routine activities immediately. In our 5- to 10-year review, three patients developed hydrocephalus and required placement of a ventriculoperitonal shunt.[13]

All new or worsened postradiosurgery deficits occurred within 28 months and no patient described a new neurological problem after the 3rd year. Normal facial nerve function was preserved in $79 \%$ of patients who could be evaluated and no patient with normal function (House-Brackmann Grade 1) developed complete facial weakness (Grade 6) after radiosurgery. The most important factor for facial neuropathy was higher tumor margin dose $(\mathrm{p}<0.003)$ and longer transverse tumor diameter $(\mathrm{p}<0.003) .[5]$

Normal trigeminal nerve function was preserved in $74 \%$ of patients in the long-term assessment and no patient with an intracanalicular tumor developed facial sensory dysfunction. Tumor volume $(\mathrm{p}<0.0001)$ and margin dose $(\mathrm{p}<0.001)$ proved to be important factors in this deficit. No change in hearing grade (Gardner-Robertson) was found in 51\% of 85 patients and $47 \%$ retained useful hearing (Gardner-Robertson Grades 1 and 2). Some degree of hearing and sound recognition was preserved in $61 \%$. Dose planning using CT scans was followed by a higher rate of hearing loss.[5,13]

\section{Neurofibromatosis Type 2}

On serial imaging studies performed over a median 36-month follow-up period (range 6-120 months), we found that 16 tumors $(36 \%)$ had regressed, $28(62 \%)$ remained unchanged in size, and one (2\%) demonstrated progression. Loss of central contrast within the tumor was frequently, but inconsistently, observed and thought to reflect tumor necrosis. The one patient whose tumor demonstrated progression despite radiosurgery was treated early in our experience (1988), without the benefit of MR image planning, and ultimately underwent surgical resection. The cumulative tumor control rate was $98 \%$.

The mean clinical follow-up period was 41 months (range 6-120). No patient demonstrated improvement at clinical examination after radiosurgery. Thirty patients $(67 \%)$ maintained stable examination results and 15 patients (33\%) demonstrated clinical deterioration. Two patients (4\%) died during the follow-up period secondary to unrelated illnesses. The median Karnofsky Performance Scale score after radiosurgery was 80 . Thirty-five patients $(78 \%)$ were able to perform normal daily activities at the time of the last examination (Karnofsky Performance Scale score $>/=80$ ).

Of the 14 patients with tumors associated with useful hearing (Gardner-Robertson Grades 1 or 2) at the time of radiosurgery, six patients (43\%) demonstrated no change in hearing grade during the follow-up period. Eight other patients lost all functional hearing (defined as absent speech discrimination) at a mean 6 months after radiosurgery (range 3-15 months). The overall rate of hearing preservation in the series was 43\%. In 1992, we began to use MR imaging-guided stereotactic planning with increasing numbers of smaller isocenters. Prior to that, we were disappointed with hearing preservation results.[17] By specifically dividing the population into those patients treated prior to 1992 and those treated after 1992, the difference in hearing preservation becomes apparent (Table 2). Prior to 1992, five patients with useful hearing (Gardner-Robertson Grade 1 or 2) were treated, and they subsequently lost all speech discrimination after treatment. After 1992, nine patients with useful hearing underwent radiosurgery, and six of the patients $(67 \%)$ had hearing preservation at the time of last examination. 


\begin{tabular}{|c|c|c|}
\hline \multicolumn{3}{|c|}{$\begin{array}{c}\text { TABLE } 2 \\
\text { OVERALL HEARING PRESEFVTION IN PATIENTS WITH } \\
\text { ACOUSTIC NEUROMAS AND NF2 TREATED WITH } \\
\text { STEREOTACTK RADOSURGERV BEFORE AND AF TER } 1992\end{array}$} \\
\hline \multirow{2}{*}{$\begin{array}{l}\text { Gardner- } \\
\text { Robertson Grade }\end{array}$} & \multicolumn{2}{|c|}{ No. of Patierts } \\
\hline & Preop & Postop \\
\hline prior to 1992 & 19 & 19 \\
\hline 1 & 3 & 0 \\
\hline 2 & 2 & 0 \\
\hline 3 & 5 & 1 \\
\hline 4 & 0 & 0 \\
\hline 5 & 9 & 18 \\
\hline after 1992 & 26 & 26 \\
\hline 1 & 6 & 4 \\
\hline 2 & 3 & 2 \\
\hline 3 & 3 & 1 \\
\hline 4 & 0 & 0 \\
\hline 5 & 14 & 19 \\
\hline
\end{tabular}

Thirty-one tumors (69\%) were associated with intact facial nerve function (House-Brackmann Grade 1) at the time of radiosurgery. Normal function of the facial nerve was preserved in 22 patients $(71 \%)$. Deterioration in function was observed in nine patients (29\%) at a mean of 8 months (range 4-24 months) after radiosurgery. Of the nine patients who experienced worsened facial nerve function, three eventually improved to Grade 1, five returned to Grade 2 function, and one remained at Grade 3. The overall rate of facial nerve preservation (Grade 1 ) was $81 \%$.

Thirty-six of the treated tumors were associated with intact trigeminal nerve function. Three patients (8\%) experienced trigeminal distribution sensory loss at a mean of 5 months after radiosurgery (range 4-5 months). One patient subsequently recovered all trigeminal function, and two patients manifested residual deficits. The overall rate of trigeminal nerve preservation was $94 \%$.

\section{DISCUSSION}

\section{Tumor Resection or Radiosurgery?}

Survey responses from 541 patient members of the Acoustic Neuroma Association provided data on tumor resection between 1973 and 1983.[28] Of these patients, 62\% reported facial weakness; 84\%, eye-related problems; $38 \%$, depression; $26 \%$, sleep disturbances; and $16 \%$, speech or swallowing difficulties. More recently in a larger survey of 1579 patients who underwent resections performed between 1989 and 1994, the authors found improved results that included a 44\% rate of facial weakness, an $11 \%$ rate of cerebrospinal fluid leakage, and persistent balance problems after 1 year in $9 \%$; approximately $8 \%$ had recurrent or residual tumor on follow-up imaging studies.[29]

Since the years of those studies, even better results have been documented after resection. Samii, et al.,[26] and Gormley, et al.,[10] have found that complete tumor removal was a frequent outcome; however, neurological and systemic morbidity remained present with $1 \%$ mortality rates and cerebrospinal fluid fistula rates of $9.2 \%$ and $15 \%$, respectively. Experienced surgical teams report significant reductions in postresection complication rates. For patients with large acoustic tumors $(>3 \mathrm{~cm}$ in extracanalicular diameter) and those with progressive neurological deficits that require brainstem decompression, surgical resection (total or subtotal) is the preferred option. We believe that a complete 
resection should be performed in such patients, if possible, but not at the expense of lost neurological function. Stereotactic radiosurgery can be considered for patients with intracanalicular, small-, or medium-sized acoustic tumors because most of these patients do not have a rapidly progressive neurological syndrome. The initial symptoms caused by most acoustic tumors are not improved by resection.[23]

The long-term effects of both resection and radiosurgery must be documented to assist physician and patient decision-making. Surprisingly little information has been published based on long-term imaging studies after resection. Cerullo, et al.,[2] noted a 10\% recurrence rate by 10 years following resection. Mazzoni, et al.,[19] reported their series of more than 100 patients in whom they attempted hearing preservation; the overall tumor recurrence rate was $8.1 \%$. Post, et al.,[24] found that four (7\%) of 56 patients underwent an incomplete resection in their attempted hearing-preservation series and that three developed regrowth within 3 years. In the largest series, Samii, et al.,[26] reported a complete resection in $98 \%$ of patients and found later recurrence in six of 880 who did not have NF2. In our radiosurgery series, $98 \%$ of patients required no further surgery, and $94 \%$ had imaging confirmation of persistent tumor control. Similar results were found for patients with solitary or NF2 tumors. After 3 years of postradiosurgery follow up, regardless of tumor type, we found no instance of delayed tumor enlargement; most patients demonstrated further tumor reduction. Nevertheless we continue to follow all our patients with imaging studies every 2 to 3 years once they pass the 8-year postradiosurgery mark.

We now believe that all patients with newly diagnosed, residual, or recurrent acoustic tumors $(<3 \mathrm{~cm}$ in extracanalicular diameter) are suitable candidates for radiosurgery. In radiobiology studies, analysis of results has shown that the doses used caused tumor regression in a human xenograft model.[18] Patients with larger tumors are not as good candidates because of the dose reduction necessary to reduce the rate of adverse radiation-related effects. In our first 3 years experience, we accepted elderly patients, those whose concomitant medical problems contraindicated resection, patients with residual or recurrent tumors after resection, and patients with preserved hearing function. By 1991 we began to offer radiosurgery to all patients with acoustic tumors regardless of age, surgical history, or symptoms. We continued to observe older patients (> 70 years of age) with small and minimally symptomatic tumors and recommended management only for imaging-defined tumor growth or progressive symptoms.[1,27,30]

\section{Evolution of Technique and Effect on Cranial Nerve Outcomes}

Refinements in technique followed a continued review of results. When we found an approximate $30 \%$ facial or trigeminal nerve morbidity rate, albeit delayed and usually mild, we reduced the tumor margin dose by an average of $2 \mathrm{~Gy}$. Our initial attempts to preserve useful hearing in patients with NF2 who underwent radiosurgery proved unsuccessful. For that reason, we more cautiously performed radiosurgery in patients with good hearing, doing so only after tumor growth had been documented and after the patient learned lip reading or signing if the tumor was on their only hearing side.

In 1991 we began to use MR imaging-based stereotactic planning because CT-based planning did not clearly show the intracanalicular portion of the tumor. With MR imaging, we could visualize the tumor and regional neural structures in greater detail. This facilitated the use of multiple, small irradiation isocenters for more conformal radiosurgery. With this type of radiosurgery, cranial nerve morbidity rates dropped precipitously, with facial and trigeminal nerve side effects below $7 \%$ for extracanalicular tumors and below $2 \%$ for intracanalicular tumors. Similarly, our analysis of hearing preservation in patients with 
NF2 showed significant gains. Whereas no patient maintained hearing at a Gardner-Robertson Grade 1 or 2 level before 1992 (zero of five patients), six of nine patients did so after 1992, with improved radiosurgery techniques. At the same time, the high rate of tumor growth control was maintained.

\section{CONCLUSIONS}

Radiosurgery is a surgical procedure associated with minimal functional morbidity that allows the patient to return rapidly to their activities. Most tumors regress in volume, as seen with extended follow up, and tumor regrowth after radiosurgery is rare and appears to happen early. Similarly, cranial neuropathy or other neurological symptoms after irradiation occur within the first few years and are usually mild and transient. Useful hearing preservation in patients with NF2 appears to be an attainable goal with more sophisticated radiosurgery techniques. We anticipate the increased use of stereotactic radiosurgery in patients with vestibular schwannomas as more and more tumors are identified at smaller sizes.

\section{Acknowledgments}

The authors thank the staff of the Center for Image-Guided Neurosurgery at the University of Pittsburgh. Click here to view commentary on this article.

\section{References}

1. Bederson JB, von Ammon K, Wichmann W, et al: Conservative treatment of patients with acoustic tumors. Neurosurgery 28:646-651,1991

2. Cerullo LJ, Grutsch JF, Heiferman K, et al: The preservation of hearing and facial nerve function in a consecutive series of unilateral vestibular nerve schwannoma surgical patients. Surg Neurol 39:485-493,1993

3. Foote RL, Coffey RJ, Swanson J, et al: Stereotactic radiosurgery using the gamma knife for acoustic neuromas. Int J Radiat Oncol Biol Phys 32:1153-1160,1995

4. Flickinger JC, Kondziolka D, Lunsford LD: Dose and diameter relationships for facial, trigeminal, and acoustic neuropathies following acoustic neuroma radiosurgery. Radiother Oncol 41:215-219,1996

5. Flickinger JC, Kondziolka D, Pollock BE, et al: Evolution in technique for vestibular schwannoma radiosurgery and effect on outcome. Int J Radiat Oncol Biol Phys 36:275-280, 1996

6. Flickinger JC, Lunsford LD, Coffey RJ, et al: Radiosurgery of acoustic neurinomas. Cancer 67:345-353, 1991

7. Flickinger JC, Lunsford LD, Linskey ME, et al: Gamma knife radiosurgery for acoustic tumors: multivariate analysis of four year results. Radiother Oncol 27:91-98, 1993

8. Flickinger JC, Lunsford $\mathrm{LD}, \mathrm{Wu} \mathrm{A}$, et al: Treatment planning for gamma knife radiosurgery with multiple isocenters. Int J Radiat Oncol Biol Phys 18:1495-1501, 1990

9. Gardner G, Robertson JH: Hearing preservation in unilateral acoustic neuroma surgery. Ann Otol Rhinol Laryngol 97:55-66, 1988 
10. Gormley WB, Sekhar LN, Wright DC, et al: Acoustic neuromas: results of current surgical management. Neurosurgery 41:50-60, 1997

11. House JW, Brackmann DE: Facial nerve grading system. Otolaryngol Head Neck Surg 93:146-147, 1985

12. Kondziolka D, Dempsey PK, Lunsford LD, et al: A comparison between magnetic resonance imaging and computed tomography for stereotactic coordinate determination. Neurosurgery 30:402-407, 1992

13. Kondziolka D, Lunsford, McLaughlin M, et al: Long term outcomes after acoustic tumor radiosurgery. N Engl J Med (In press)

14. Lalwani A, Butt FY, Jackler R, et al: Delayed onset facial nerve dysfunction following acoustic neuroma surgery. Am J Otol 16:758-764, 1995

15. Linskey ME, Flickinger JC, Lunsford LD: Cranial nerve length predicts the risk of delayed facial and trigeminal neuropathies after acoustic tumor stereotactic radiosurgery. Int J Radiat Oncol Biol Phys 25:227-233, 1993

16. Linskey ME, Lunsford LD, Flickinger JC: Neuroimaging of acoustic nerve sheath tumors after stereotaxic radiosurgery. AJNR 12:1165-1175, 1991

17. Linskey M, Lunsford LD, Flickinger JC: Tumor control after stereotactic radiosurgery in neurofibromatosis patients with bilateral acoustic tumors. Neurosurgery 31:829-839, 1992

18. Linskey ME, Martinez AJ, Kondziolka D, et al: The radiobiology of human acoustic schwannoma xenografts after stereotactic radiosurgery evaluated in the subrenal capsule of athymic mice. J Neurosurg 78:645-653, 1993

19. Mazzoni A, Calabrese V, Moschini L: Residual and recurrent acoustic neuroma in hearing preservation procedures: neuroradiologic and surgical findings. Skull Base Surg 6:105-112, 1996

20. Norén G, Arndt J, Hindmarsh T: Stereotactic radiosurgery in cases of acoustic neurinoma: further experiences. Neurosurgery 13:12-22, 1983

21. Ogunrinde OK, Lunsford LD, Flickinger JC, et al: Cranial nerve preservation after stereotactic radiosurgery for small acoustic tumors. Arch Neurol 52:73-79, 1995

22. Ogunrinde OK, Lunsford LD, Flickinger JC, et al: Stereotactic radiosurgery for acoustic nerve tumors in patients with useful preoperative hearing: results at 2-year follow-up examination. $\mathbf{J}$ Neurosurg 80:1011-1017, 1994

23. Pollock BE, Lunsford LD, Kondziolka D, et al: Outcome analysis of acoustic neuroma management: a comparison of microsurgery and stereotactic radiosurgery. Neurosurgery 36:215-229, 1995

24. Post KD, Eisenberg MB, Catalano PJ: Hearing preservation in vestibular schwannoma surgery: what factors influence outcome? J Neurosurg 83:191-196, 1995

25. Rowed DW, Nedzelski JM: Hearing preservation in the removal of intracanalicular acoustic neuromas via the retrosigmoid approach. J Neurosurg 86:456-461, 1997 
26. Samii M, Matthies C: Management of 1000 vestibular schwannomas (acoustic neuromas): Surgical management and results with an emphasis on complications and how to avoid them. Neurosurgery 40:11-23, 1997

27. Samii M, Tatagiba M, Matthies C: Acoustic neurinoma in the elderly: factors predictive of postoperative outcome. Neurosurgery 31:615-620, 1992

28. Wiegand DA, Fickel V: Acoustic neuroma--the patients perspective: subjective assessment of symptoms, diagnosis, therapy, and outcome in 541 patients. Laryngoscope 99:179-187, 1989

29. Wiegand DA, Ojemann RG, Fickel V: Surgical treatment of acoustic neuroma (vestibular schwannoma) in the United States: report from the Acoustic Neuroma Registry. Laryngoscope 106:58-66, 1996

30. Yamamoto M, Hagiwara S, Ide M, et al: Conservative management of acoustic neurinomas: prospective study of long-term changes in tumor volume and auditory function. Min Invas Neurosurg 41:86-92, 1998

Manuscript received July 9, 1998.

Accepted in final form August 31, 1998.

Dr. Kondziolka was supported by National Institutes of Health Grant No. K08 NS01723.

Address reprint requests to: Douglas Kondziolka, M.D., Department of Neurological Surgery, Suite B-400, University of Pittsburgh Medical Center, 200 Lothrop Street, Pittsburgh, Pennsylvania 15213. email: kondziol@neuronet.pitt.edu. 